\title{
Fractional Order Control of a DC Motor with Load Changes
}

\author{
${\text { Cosmin } \text { Copot }^{1} \text {, Cristina Muresan }}^{2}$, Clara M. Ionescu $^{2}$, Robin De Keyser ${ }^{1}$ \\ ${ }^{1}$ Department of Electrical energy, Systems and Automation, Ghent University \\ Gent, Belgium \\ ${ }^{2}$ Technical University of Cluj-Napoca, Department of Automation \\ Cluj-Napoca, Romania
}

\begin{abstract}
This paper investigates the robustness of a fractional-order controller against the load changes of a DC motor. The gains and time constants of the DC motor are modified by means of a change in the brake. Two different setups of a DC motor, one with $25 \%$ brake and the other with $50 \%$ brake are considered in the experimental evaluation. The closed-loop performances of the fractional-order controller are compared with integer-order controller using the same performance criteria and the same tuning algorithm. Both controllers were designed based on time domain specifications. The experimental results show that the fractional-order controller outperforms the classical controller under nominal conditions as well as under gain variations situation.
\end{abstract}

\section{INTRODUCTION}

Mechatronic systems are governed by many effects residing in different engineering fields, covering problems form mechanical, electrical, pneumatic, thermal disciplines, comprising various technological components. Thus, these types of systems are usually characterized by significant nonlinearities and tight performance specifications [1]. Due to these features, the main challenge in the design and analysis of mechatronics systems consists in an accurate modelling [2], [3]. Whenever such a model is difficult to be obtained precisely, the controller needs to be properly designed in order to compensate for the modelling uncertainties and to preserve the closed-loop performance at various operating points. Numerous control algorithms have been proposed to deal with nonlinear dynamics of the mechatronic systems. For linear mechatronic systems, the proportional-integral-derivative (PID) controller is often used owing to its simple structure and robustness [4]. Another approach in dealing with mechatronic systems challenges is the fractional-order (FO) control strategies. One of the most common applications in all mechatronic domains is the control of DC motors.

The control of DC motors has been the interest of many researchers, due to the wide variety of applications that require the use of different types of DC motors [5], [6], [7]. The controllers designed for these DC motors range from simple traditional PIDs to advanced control algorithms, among which fractional-order control has been gaining more and more popularity [8], [9], [10], [11].

Fractional calculus has been used relatively recently in modeling and control applications [12]. The attractiveness of the fractional-order PID controllers resides in their potential to increase the closed-loop performance and robustness of the closed-loop system, due to the extra tuning parameters available, as compared to the conventional controller. With fractional-order controllers, the order of differentiation and integration may be used as supplementary tuning parameters and thus more specifications can be fulfilled at the same time, including the robustness to plant uncertainties, such as gain and time constant changes [12], [13], [14].

In general, frequency domain tuning of the fractional-order controllers is preferred using optimization routines to yield the final solutions. The performance criteria are frequently specified in terms of gain crossover frequency, phase crossover frequency, phase margin, gain margin, and robustness to openloop gain variations [15], [16].

In this paper a fractional calculus based control strategy for speed control of a DC motor with load changes is presented. The relevance of the paper to the research field consists in the simplicity of the approach, yet yielding a robust controller that can meet the performance specifications for significant load changes. The robustness of the fractional-order PI controller and its performances are compared against an integer-order PI controller. In order to evaluate the robustness of the controllers a change in the motor loading unit is considered for the conducted experiments. Due to the change in the brake unit, the gain and time constant of the system are also modified. The performances of both classical integer-order approach and fractional-order approach are analyzed through simulations and real-time experiments. The control design method and the application are kept simple, yet effective to illustrate basic time domain and frequency domain concepts. The experimental results revealed better performances of the fractional approach in comparison with the classical one.

The paper is structured as follows: Section II presents the tuning procedure for a fractional-order PI controller. Section III presents the description of a DC motor as well as a tuning example for the control of the speed of a DC motor. Section IV presents the simulation and real-time results of the conducted experiments, while the last section includes the conclusions.

\section{DESIGN OF A ROBUST FRACTIONAL-ORDER PI CONTROLLER}

Fractional calculus represents the generalization of the integration and differentiation to an arbitrary order. The Laplace 


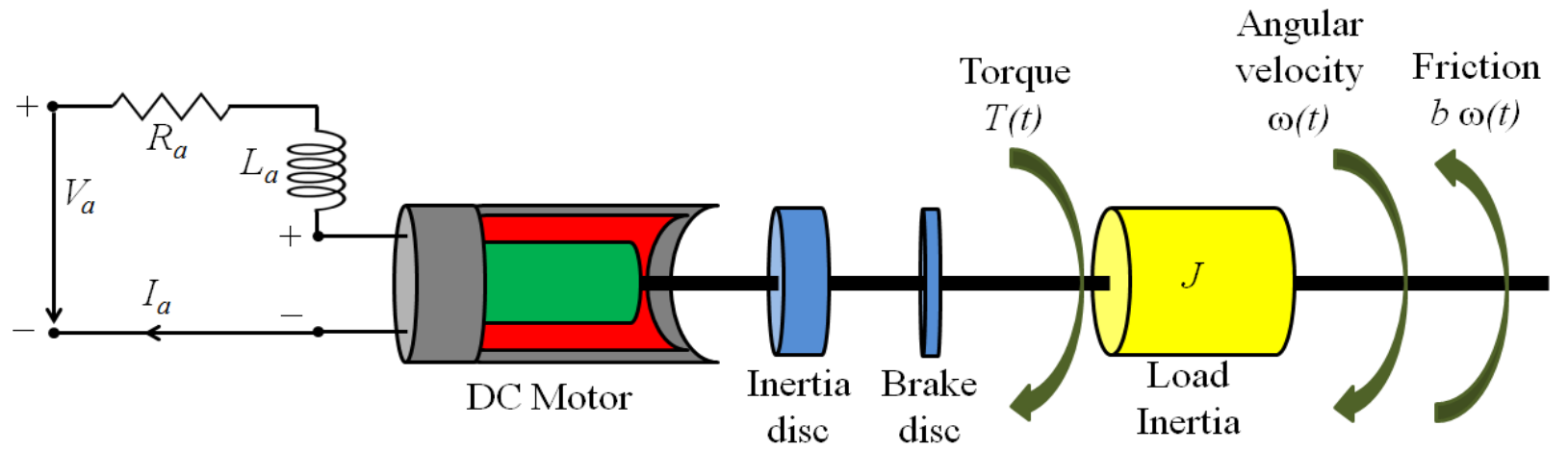

Fig. 1. General model of a DC motor

transform of the fractional-order operators are [12]:

$$
\mathrm{L}\left\langle\mathrm{I}^{\alpha} f(t)\right\rangle=s^{-\alpha} F(s)
$$

for the fractional-order integral, while for the fractional-order derivative, the equation is:

$$
\mathrm{L}\left\langle\mathrm{D}^{\alpha} f(t)\right\rangle=s^{\alpha} F(s)
$$

with $\alpha \in(0,1)$.

The transfer function of the FO-PI controller is given as:

$$
H_{F O-P I}(s)=k_{p}\left(1+\frac{k_{i}}{s^{\mu}}\right)
$$

where $k_{p}$ and $k_{i}$ are parameters of the fractional-order PI controller, while $\mu$ represents the fractional order.

The design of the FO-PI controller is usually based on a phase margin and a gain crossover condition, to which a third criteria may be added in order to uniquely determine the three controller parameters. In order to tune the fractionalorder PI controller, the open-loop transfer function needs to be computed as:

$$
H_{\text {open-loop }}(s)=H_{F O-P I}(s) H_{P}(s)
$$

where $H_{P}(s)$ is the process to be controlled. The tuning of the FO-PI controller implies the computation of the three parameters $k_{p}, k_{i}$ and $\mu$ according to three performance specifications imposed. The gain crossover frequency $-\omega_{c g}$ - implies that the modulus of the open-loop transfer function obeys the following:

$$
\left|H_{\text {open-loop }}\left(j \omega_{g c}\right)\right|=1
$$

while the phase margin $-\varphi_{m}$ - specification sets a condition upon the phase of the open-loop system at the gain crossover frequency, mathematically written as:

$$
\angle H_{\text {open-loop }}\left(j \omega_{g c}\right)=-\pi+\varphi_{m}
$$

The performance specifications given above may be rewritten as:

$$
\left|H_{F O-P I}\left(j \omega_{g c}\right)\right|=\frac{1}{\left|H_{P}\left(j \omega_{g c}\right)\right|}
$$

$$
\angle H_{F O-P I}\left(j \omega_{g c}\right)=-\pi+\varphi_{m}-\angle H_{P}\left(j \omega_{g c}\right)
$$

which can be further detailed as:

$$
\begin{gathered}
\left|k_{p}\left[1+k_{i} \omega_{g c}^{-\mu}\left(\cos \frac{\pi \mu}{2}-j \sin \frac{\pi \mu}{2}\right)\right]\right|=\frac{1}{\left|H_{p}\left(j \omega_{g c}\right)\right|} \\
\angle\left[1+k_{i} \omega_{g c}^{-\mu}\left(\cos \frac{\pi \mu}{2}-j \sin \frac{\pi \mu}{2}\right)\right]= \\
=-\pi+\varphi_{m}-\angle H_{P}\left(j \omega_{g c}\right)
\end{gathered}
$$

Since the FO-PI controller has three independent parameters, these can be adequately tuned to meet three performance specifications. Thus apart from imposing a certain gain crossover frequency and a certain phase margin, which naturally imply a certain settling time and overshoot, a third condition may be added to the design problem. This third condition can refer to high frequency noise rejection, good output disturbance rejection, robustness to variations in the gain of the plant, etc [17].

\section{CASE STUdy: DC MOTOR SPEED CONTROL USING FO-PI CONTROLLER}

DC motors are amongst the most largely used components both academic and industrial applications. An essential feature of any position or speed control system is an electric motor with some associated power supply and amplifier stage to control power input to the motor in response to a lower level control signal. In order to adequately tune the FO-PI controller as indicated in section II, the transfer function of the studied DC motor needs to be determined. A general model of the DC motor is shown in Figure 1.

The applied voltage $V_{a}$, which is the manipulated variable, will control the position $\theta(t)$, which is the controlled variable. For the speed control, the controlled variable is the angular velocity $\omega(t)$ and the transfer function has the form in [18]:

$$
P_{D C \_ \text {motor }}(s)=\frac{\omega(s)}{V_{a}(s)}=\frac{K_{m}}{\left(L_{a} s+R_{a}\right)(J s+b)+K_{b} K_{m}}
$$

However, for many DC motors the time constant of the armature $\tau_{a}=\frac{L_{a}}{R_{a}}$ is negligible and therefore the model can 
be simplified to:

$$
\begin{aligned}
P_{D C \_ \text {motor }}(s) & =\frac{K_{m}}{R_{a}(J s+b)+K_{b} K_{m}}=\frac{\frac{K_{m}}{R_{a} b+K_{b} K_{m}}}{\tau s+1}= \\
& =\frac{K_{D C} \text { motor }}{\tau s+1}
\end{aligned}
$$

where $\tau=\frac{R_{a} J}{R_{a} b+K_{b} K_{m}}$ and $K_{D C \_ \text {motor }}=\frac{K_{m}}{R_{a} b+K_{b} K_{m}}$.

The transfer function from position $\theta(t)$ as output (controlled variable) to armature voltage $V_{a}$ as input (manipulated variable) will be:

$$
P_{D C \_m o t o r \_p o s}(s)=\frac{\theta(s)}{V_{a}(s)}=\frac{K_{D C \_ \text {motor }}}{s(\tau s+1)}
$$

The identification of the system was done based on a PRBS (pseudo random binary signal) signal. To generate this PRBS signal, the following command is used in Matlab:

- idinput(127, 'PRBS', [0 1/1], [-1 1]).

By using the Prediction Error Method (PEM) for identification [19], the system's model is defined. The transfer function of the DC motor voltage-speed, with $25 \%$ brake, was identified to be:

$$
P_{D C \_ \text {motor_1 }}(s)=\frac{-0.25}{(1.45 s+1)}
$$

A second transfer function for the DC motor speed, considering $50 \%$ brake, was identified as well:

$$
P_{D C \_m o t o r \_2}(s)=\frac{-1}{1.7 s+1}
$$

The gains and time constants of the DC motor are modified due to the change in the brake. The motor loading unit (brake unit and inertia disc) consists of an eddy current brake and an inertia disc which can be mounted on the armature shaft extension. The brake has the approximate speed/torque at 1000 rpm as shown on Figure 2. For other speeds the torque is proportional to the speed relative to the $1000 \mathrm{rpm}$. The inertia moment of the inertia disc is about $0.001 \mathrm{Kg}^{*} \mathrm{~m}^{2}$.

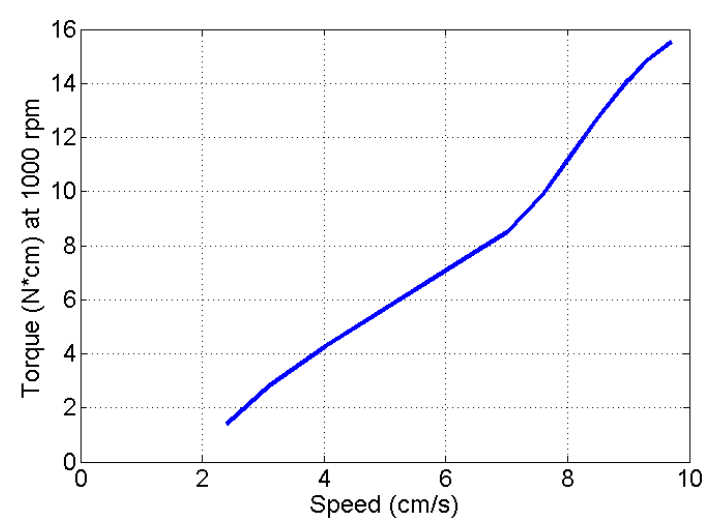

Fig. 2. Approximate brake characteristics at $1000 \mathrm{rpm}$

If the inertia of the motor is increased the transient response is slowed down and hence with additional inertia the maximum acceleration and deceleration are reduced. The motor responds fairly rapidly to the step input and its rise in speed follows an exponential path when no discs are mounted on the output shaft (Figure 3(a)). When the input falls to zero the deceleration is due almost entirely to brush friction and is linear. If the inertia of the brake disc is added the response time is increased (Figure 3(b)). Using the inertia disc, the response time is greatly increased and the motor only just stop before the step input is re-applied (Figure 3(c)). The added inertia has little effect upon the response time, but the time for the motor to stop is much reduced.

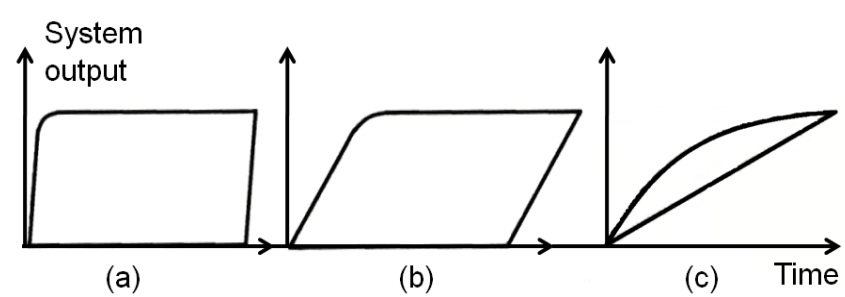

Fig. 3. The variation in the DC motor characteristic when (a) no disc is mounted on the shaft; (b) brake disc is mounted; (c) inertia disc is mounted

To design the FO-PI controller, the $P_{D C \_ \text {motor_1 }}(s)$ transfer function was considered as being the nominal one. The $P_{D C \_}$motor_2 $(s)$ transfer function is considered as a uncertainty. While the time constant does not change significantly, the gain of the DC motor transfer function changes substantially. Hence, apart from setting a certain settling time and overshoot by imposing the gain crossover frequency and phase margin, a robust controller especially to gain variations needs to be computed. As a consequence, to the previously defined two performance specifications, a third condition is appended, that refers to the robustness against gain uncertainties:

$$
\left.\frac{d\left(\angle H_{\text {open-loop }}(j \omega)\right)}{d \omega}\right|_{\omega=\omega_{g c}}=0
$$

also written as:

$$
\begin{aligned}
& \frac{d\left(\angle\left[1+k_{i} \omega_{g c}^{-\mu}\left(\cos \frac{\pi \mu}{2}-j \sin \frac{\pi \mu}{2}\right)\right]\right)}{d \omega_{g c}}= \\
& =-\frac{d\left(\angle P_{D C} \text { motor }_{1}\left(j \omega_{g c}\right)\right)}{d \omega_{g c}}
\end{aligned}
$$

The condition in (16) imposes a flat phase around the gain crossover frequency. For open-loop gain changes, the gain crossover frequency varies around the nominal value. However a flat phase around that value, will ensure a constant phase margin, equal to the one imposed in the design specifications, despite the variations in the gain crossover frequency, thus assures the robustness against gain modeling errors or uncertainties.

The performance specifications regarding the gain crossover frequency and phase margin are: $\omega_{c g}=1.5$ and $\varphi_{m}=60^{\circ}$. Such performance specifications allow for a 2 second settling time and a $5 \%$ overshoot. Using graphical methods, two curves for the $k_{i}$ parameter as a function of the fractional order $\mu$ are plotted as indicated in Figure 4. The intersection of the two curves yields the solution for $k_{i}$ and $\mu$ as resulting from (10) and (17). The final values, $k_{i}=2.28$ and $\mu=0.89$, are then 
used to compute the value for the third parameter $k_{p}$ using (9): $k_{p}=1.37$. Thus, the FO-PI controller was obtained as:

$$
H_{F O \_P I}(s)=1.37+\frac{2.28}{s^{0.89}}
$$

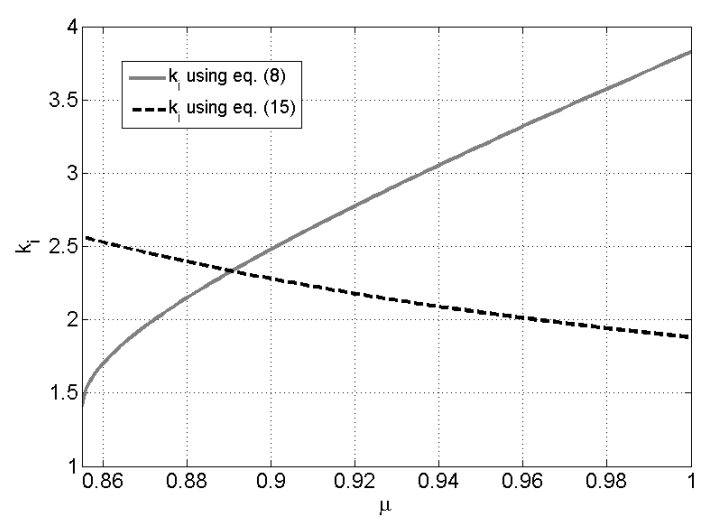

Fig. 4. Graphical selection of the fractional order PI parameters $k_{i}$ and $\mu$

To test the controller, prior to the actual implementation, a Simulink benchmark was created in which the previously determined FO-PI controller was implemented in its discrete form. To obtain the discrete approximation of the FO-PI controller the 9th order Tustin recursive method with a sampling period of 0.2 seconds was chosen.

\section{RESUlTS AND Discussion}

In this section, a part of the simulation and experimental results that were conducted in order to validate the fractional order controllers are presented.

Hence, a discrete time version of the controllers need to be developed for the final implementation. The equivalent discrete-time formulation (for sampling time $0.2 \mathrm{~s}$ ) of the identified model for DC motor voltage-speed with $25 \%$ brake is given by:

$$
P_{D C \_ \text {motor_1 }}(z)=\frac{0.032}{z-0.87}
$$

\section{A. Simulation Results}

Before to implement and evaluate the performance of the controllers on the real-time setup, a Simulink model has been designed to test and validate the controllers. The simulation results for fractional-order feedback loop by using the Simulink model are depicted in Figures 5, 6, 7. The fractional-order PI controller is compared with the integer-order PI controller. The integer-order PI controller has been designed using the same performance specifications and the same tuning algorithm as for the fractional controller. Hence, $\mu=1$ and the tuning parameters are: $k_{p}=1.23$ and $k_{i}=2.41$. As observed from the simulation results, the fractional-order controller outperforms the classical controller.

Figure 5 shows the simulation results considering the nominal case, when the DC motor transfer function is represented by $P_{D C \_}$motor_1 $(s)$, as well as the uncertain case, when the DC motor transfer function is given by $P_{D C}$ motor $22(s)$. The results concerning the nominal case, the DC motor with $25 \%$ brake, show that there is a $4.5 \%$ overshoot, while the settling time is 2 seconds, as expected from the required performance criteria. The comparison of these results with those obtained when considering a $17.25 \%$ change in the DC motor time constant and $300 \%$ change in the DC motor gain demonstrate the robustness of the designed controller. In the uncertain case, when the DC motor works with a $50 \%$ brake, the overshoot is $18.5 \%$, while the settling time is 0.4 seconds. Hence, we can conclude that the controller is very robust given the dramatic changes in the gain. .

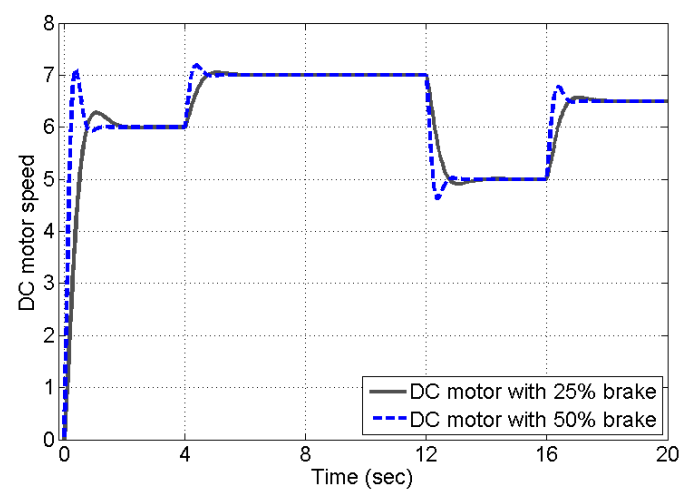

Fig. 5. Simulations results considering FO-PI controller with $25 \%$ brake (nominal case) and $50 \%$ brake (uncertain case)

Next, the results with the FO-PI controller are compared with an integer-order PI controller. As observed from the simulation results given in Figure 6 where only the nominal case is considered, the fractional-order controller slightly outperforms the classical controller. To design the PI controller, the $k_{i}$ parameter can be determined using either one of the conditions specified in (10) or (17). In order to make the integer-order PI controller more robust, (17) was used to compute the final value for parameter $k_{i}$. Thus the phase margin is no longer a tuning criteria and (8) is no longer valid. The tuning of the PI controller using (17) was selected since for the FO-PI controller a value $\mu=0.89$ for the fractional order was obtained as being the optimum value that meets both performance specifications (10) and (17). Since $\mu=0.89$ is close to unity, it is expected that the phase margin for $\mu=1$ does not decrease drastically as compared to the imposed value of $60^{\circ}$. For the integer-order PI controller, $\mu=1$ would then ensure a high enough phase margin, thus making it more convenient to determine the $k_{i}$ parameter based on the robustness condition. Figure 6 shows that under nominal conditions, the overshoot is increased, while the corresponding phase margin is in fact less than the one imposed for the design of the FO-PI controller.

To test the robustness of the integer-order PI controller, the uncertain case is considered, with the DC motor working under $50 \%$ brake. The simulation results are given in Figure 7 . The 


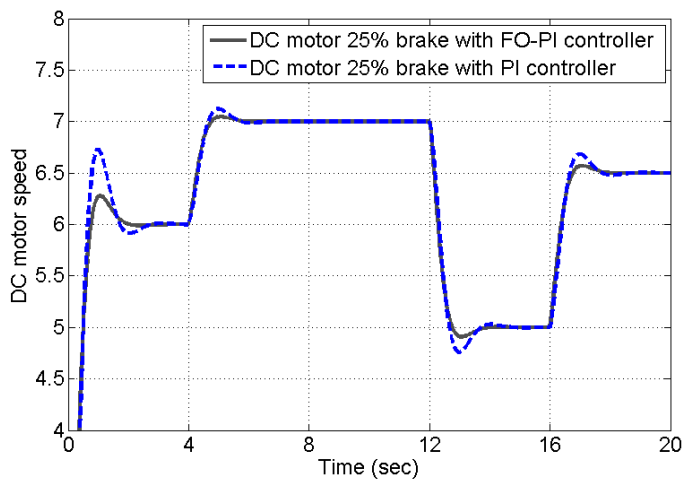

Fig. 6. Comparative simulation results for DC motor speed control with $25 \%$ brake using integer-order and fractional-order PI controllers

results considering a $50 \%$ brake show that the FO-PI controller achieves better closed-loop performance even in the case of gain variations as compared to the integer-order PI controller.

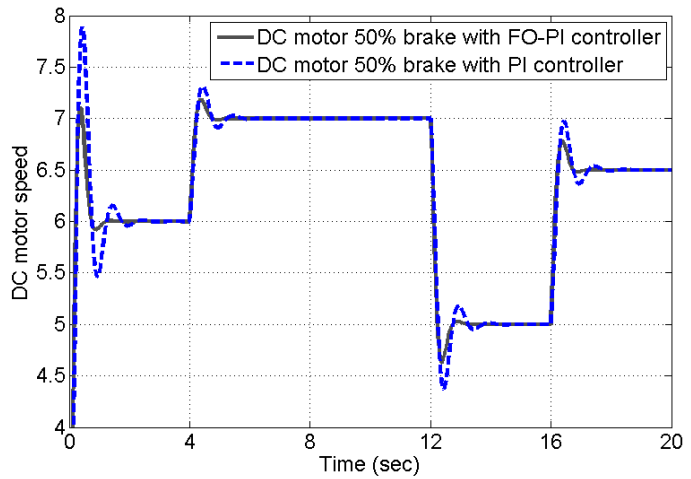

Fig. 7. Comparative simulation results for DC motor speed control with $50 \%$ brake using integer-order and fractional-order PI controllers

Table 1 presents the values obtained for the overshoot and settling time considering both FO-PI and PI controllers, under nominal as well as uncertain situations. It is clear from this table that the FO-PI controller achieves far better performance results than the PI controller both under nominal conditions, but especially under gain variations.

TABLE I

Closed-LOOP PERFORMANCE RESULTS OBTAINED WITH THE DESIGNED FO-PI AND PI CONTROLLERS

\begin{tabular}{c|cc|cc} 
Controller & \multicolumn{2}{|c|}{ Overshoot } & \multicolumn{2}{c}{ Settling time } \\
\hline \hline & $25 \%$ brake & $50 \%$ brake & $25 \%$ brake & $50 \%$ brake \\
\hline FO-PI & $4.5 \%$ & $18.5 \%$ & $2 \mathrm{sec}$ & $0.4 \mathrm{sec}$ \\
PI & $12 \%$ & $31.5 \%$ & $2 \mathrm{sec}$ & $2 \mathrm{sec}$ \\
\hline
\end{tabular}

To validate the results obtained in Simulink, real experiments were conducted using both controllers under $25 \%$ brake, as well as $50 \%$ brake.

\section{B. Experimental Results}

The performance of the PI controllers has also been evaluated in the real-time application using the set-up depicted in Figure 8. After the system reaches steady state, different changes in setpoint were applied. Again, the experimental results from Figures 9 and 10 show that the fractional-order controller outperforms the integer-order PI controller. If for the setpoint tracking the superiority of the fractional controller is slightly visible, in case of control effort the fractional-order PI is clearly superior to the classical controller. A comparison of the closed-loop performance of the fractional-order PI controller when considering the DC motor with $25 \%$ brake and $50 \%$ brake, respectively, is illustrated in Figure 11.

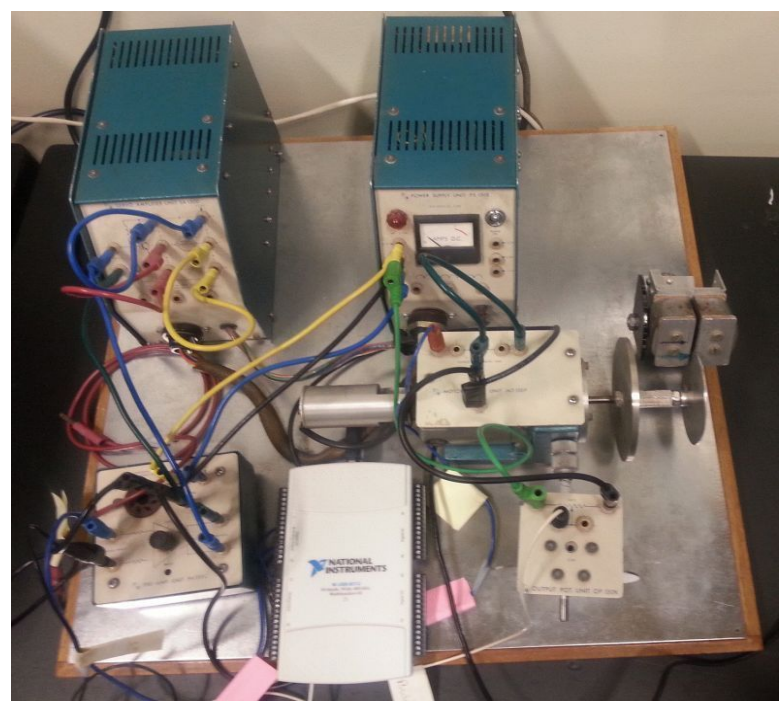

Fig. 8. The real set-up configuration for the DC motor
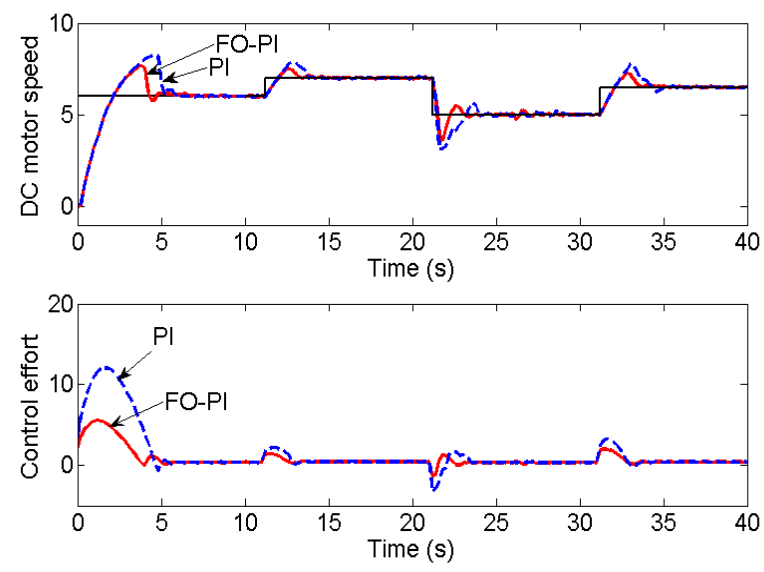

Fig. 9. Comparative real-time results for DC motor speed control with $25 \%$ brake using fractional-order and integer-order PI controllers

The simulation and experimental results reveal that fractional-order controller obtain better performances when dealing with this type of process in comparison with classical 

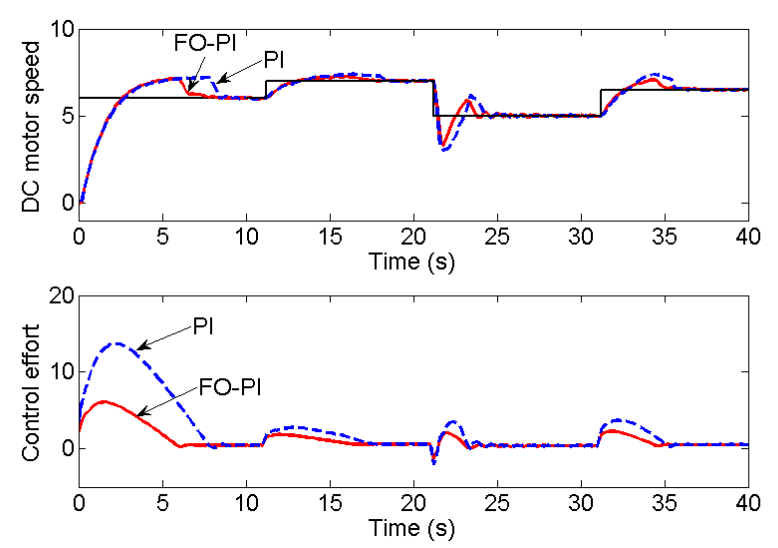

Fig. 10. Comparative real-time results for DC motor speed control with $50 \%$ brake using fractional-order and integer-order PI controllers
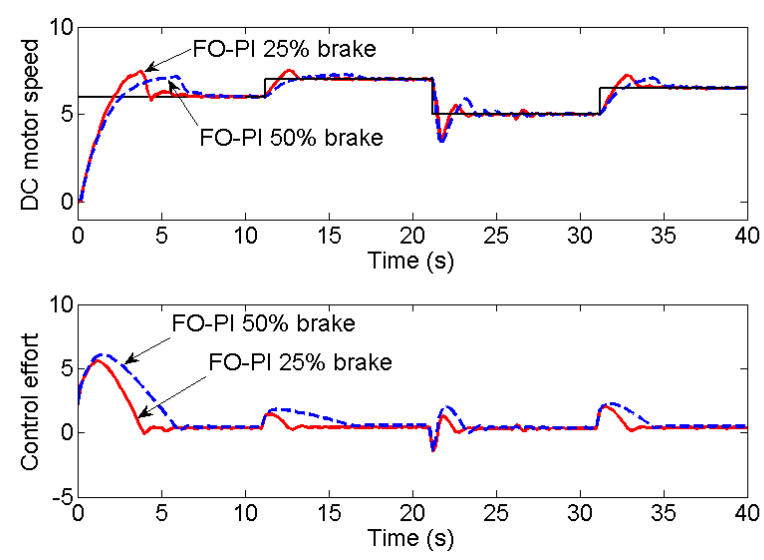

Fig. 11. Comparative real-time results for DC motor speed control with $25 \%$ and $50 \%$ brake using fractional-order PI controller

PI controller. An important issue of the real-time processes is to have a faster convergence of the system with less control effort. Analyzing the experimental results it can be notice that the fractional-order controller is robust to the load changes of the DC motor and obtain good performances without great control effort.

\section{CONCLUSiON}

In this paper, an illustrative example of a fractional-order controller for speed control of a DC motor with load changes is presented. The robustness of the fractional-order controller has been evaluated by changing the gains and time constant of the DC motor due to the change in the brake unit. The experimental results are performed using a fractional-order PI controller and the output performances are compared with integer-order PI controller. Both controllers were designed using the same tuning algorithm. Due the flexibility of the fractional order parameters, the system can fulfill more specifications and thus becomes more robust to changes in the dynamics. The experimental results show that the fractional-order controller outperforms the classical integer-order controller. On the other side the design of a fractional-order controller implies computational complexity and also the implementation itself may require additional effort.

\section{ACKNOWLEDGMENT}

This work was partially supported by a grant of the Romanian National Authority for Scientific Research, CNCS UEFISCDI, project number PN-II-RU-TE-2012-3-0307.

\section{REFERENCES}

[1] S. Di Cairano, A. Bemporad, I. Kolmanovsky, D. Hrovat, Model predictive control of nonlinear mechatronic systems: An application to a magnetically actuated, 2nd IFAC Conference on Analysis and Design of Hybrid Systems, 2, Italy, 2006.

[2] M. Steinbuch, R. Merry, M. Boerlage, M. Ronde and M. van de Molengraft, Advanced motion control design. In W.E. In Levin (ed.), The Control Handbook, Control System Applications, 27-1/25. CRC Press, 2010.

[3] R. Bishop, The Mechatronics Handbook. CRC Press, 2002.

[4] K. Åström and T. Hägglund, Advanced PID control. ISA-The Instrumentation, Systems, and Automation Society, 2006.

[5] M. Steinbuch and M. Norg, Advanced motion control: An industrial perspective. European Journal Control, 4, pp. 278-293, 1998.

[6] S. Yang and J. Chou, A mechatronic positioning system actuated using a micro dc - motor - driven propeller-thruster. Mechatronics, 19, pp. 912-926, 2009.

[7] J. De Best, M. van de Molengraft and M. Steinbuch, A novel ball handling mechanism for the robocup middle size league. Mechatronics, 21(2), pp. 469-478, 2011.

[8] D. Xue, C. Zhao and Y. Chen, Fractional order pid control of a de motor with elastic shaft: A case study. In Proceedings of the 2006 American Control Conference. USA, 2006.

[9] J. Villagra, B. Vinagre and I. Tejado, Data-driven fractional pid control: application to dc motors in flexible joints. In Proceedings of the IFAC Conference on Advances in PID Control PID'12. Brescia (Italy), 2012.

[10] C. Muresan, S. Folea, G. Mois and E. Dulf, Development and implementation of an fpga based fractional order controller for a dc motor. Mechatronics, 23 (7), pp. 798-804, 2013.

[11] C. Copot, A. Burlacu, C. Ionescu, C. Lazar and R. De Keyser, A fractional order control strategy for visual servoing systems. Mechatronics, 23, pp. 848-855, 2013.

[12] C. Monje, Y. Chen, B. Vinagre, D. Xue and V. Feliu, Fractionalorder Systems and Controls, Fundamentals and Applications. Springer, London, 2010.

[13] C. Pop, C. Ionescu, R. De Keyser and E. Dulf, Robustness evaluation of fractional order control for varying time delay processes. Signal, Image and Video Processing, 6, pp. 453-461, 2012.

[14] A. Oustaloup, La commande CRONE: commande robust d'ordre non entier. Hermes, Paris, 1991

[15] C. Monje, B. Vinagre, Y. Chen and V. Feliu, On fractional $p i^{\lambda}$ controllers: some tuning rules for robustness to plant uncertainties. Nonlinear Dynam., 38, pp. 369-381, 2004.

[16] I. Podlubny, Fractional-order systems and $P I^{\lambda} D^{\mu}$ - controllers. IEEE Trans. Automat. Control., 44, pp. 208-214, 1999.

[17] C. Monje, B. Vinagre, V. Feliu and Y. Chen, Tuning and autotuning of fractional order controllers for industry applications. Control Engineering Practice, 16, pp. 798-812, 2008.

[18] R. Dorf and R. Bishop, Modern Control Systems. Addison-Wesley, New York, 2000.

[19] L. Ljung, System identification: theory for the user. Prentice-Hall, 2007. 